\title{
The Role of Evolutive Elastic Properties in the Performance of a Sheet Formed Spring Applied in Multimedia Car Industry
}

\author{
Joel Silva ${ }^{2, a}$, João Faria ${ }^{1}$, Rita Ferreira $^{1}$, Pedro Bernardo ${ }^{2}$ and J. L Alves ${ }^{1}$ \\ ${ }^{1}$ CMEMS, University of Minho Campus of Azurém, 4800-058 Guimarães, Portugal \\ ${ }^{2}$ Bosch Car Multimedia Portugal S.A., Apartado 2458, 4705-820 Braga, Portugal
}

\begin{abstract}
The manufacturing process and the behavior of a sheet formed spring manufactured from an aluminum sheet is described and investigated in this work considering the specifications for the in-service conditions. The sheet formed spring is intended to be applied in car multimedia industry to replace bolted connections. Among others, are investigated the roles of the constitutive parameters and the hypothesis of evolutive elastic properties with the plastic work in the multi-step forming process and in working conditions.
\end{abstract}

\section{Introduction}

The competitiveness of the automotive industry has led to a simultaneous increase of innovation and a more efficient costs control, which are the key to achieve better products and consequently meet strategic business goals. The tendency to use lightweight materials and innovative design concepts are usually focused on car body panels and chassis. However, this design concept should be extended to all metallic car components, even in auxiliary parts like the ones in multimedia chassis applications.

The metallic chassis of multimedia systems are usually sheet metal parts and the assembling of its components is mostly made with bolts. Bolted connections are responsible for joining the parts together, besides they must provide ground contact and ensure an electromagnetic shielding of the assembled system.

Bolted connections have several drawbacks since each bolt must be carefully and individually bolted at the right position and with a pre-determined tightening torque. Having in consideration that each chassis may contain several bolts, the screwing duration is a significant amount of time consumed during the assembling process. Because the used bolts are the selftapping type, during the screwing process occurs the production of metallic shavings, which once deposited in the printed circuit boards or other electronic components may provoke short circuit and consequently malfunctions.

The bolt nonconformity is also a problem because it makes the process repeatability very hard to ensure.

The present study aims to investigate an innovative solution for fixing a printed circuit board under a metallic chassis without bolted connections. This geometry must be capable of submitting the printed circuit board under a compressive load, to ensure the ground contact between components.

The compressive load has a key role in this geometry: it must be capable to replace the bolt's compressive load and ensure a good performance in several tests specified in automotive standards.

The main methodologies applied to evaluate and predict the material and geometry's performance are experimental tests and numerical simulations. With the rapid development of computational capacity, the finite element analysis has been widely used by scientific and industrial communities in product design and to predict material's behaviour in manufacturing process. Finite element analysis reduces the number of trials, reducing the cost of product development [1,2].

The problem investigated in this paper is to evaluate, by means of numerical simulation, the usage of a new sheet formed spring to replace bolted connections, and predict its compressive load.

The research objectives are to evaluate the effects of the manufacturing process, the evolution of the elastic properties due to the plastic work, and the hardening models, in the prediction of the proposed geometry behaviour. To evaluate these effects several numerical simulations were performed applying different parameters, and finally the main results are compared and discussed.

\section{The new sheet formed spring: geometry description}

The proposed geometry was designed having in mind the industrial problem previously addressed. This geometry is located at the cover corners of a metallic chassis, and it consists in a recess with a trapezium format where the contact between the printed circuit board and chassis will occur (see more details on Figure 1).

\footnotetext{
a Corresponding author: vieiradasilvajf@gmail.com
} 
The innovation presented in this geometry is focused on the geometry around the recess. This geometry consists of material removal in the shape of tabs and it was designed to have a similar behaviour to a spring. The geometry shown in (Figure 1) was subject of previous numerical simulations aiming to find the best geometry's shape of the tabs. The geometry selected has shown the better ratio force/displacement.

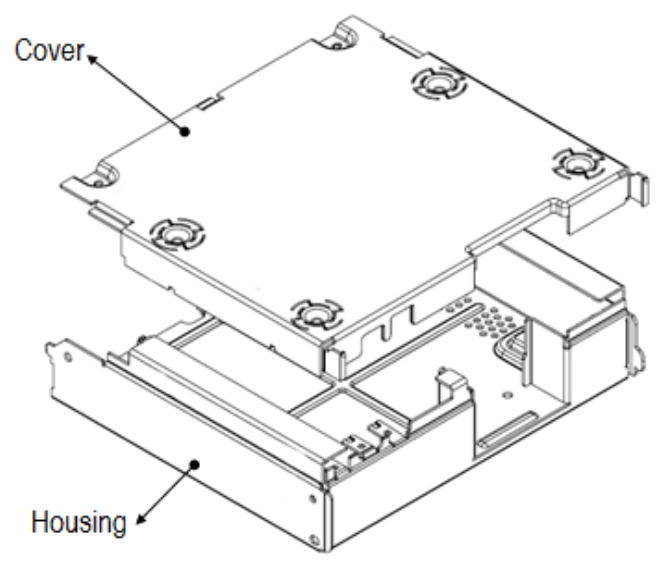

a)
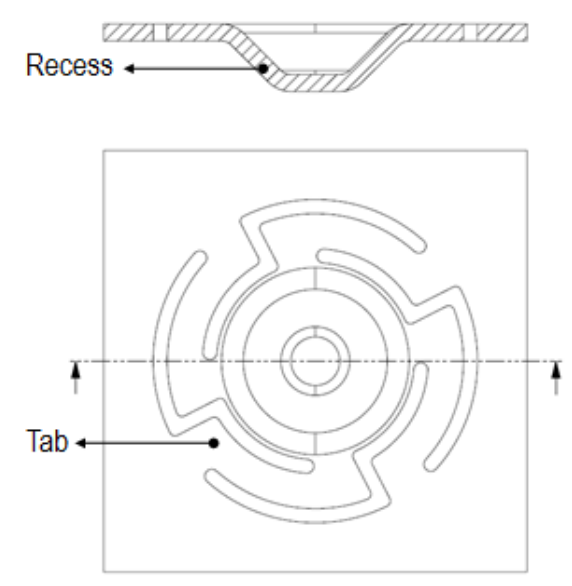

b)

Figure 1. Developed geometry to replace bolts: a) its placement in the chassis top cover, and b) detailed geometry of the sheet formed spring, comprising a recess and four tabs.

\section{Numerical modelling}

Through finite element analysis the numerical studies are an important tool allowing the shortening of several resources, such as time, money and useless prototypes.

\subsection{DD3IMP}

Deep Drawing 3D IMPlicit Code (DD3IMP) is a finite element code which is the result from continuous development and optimizations aimed for the simulation of sheet metal forming processes $[3,4]$. This FE solver considers the evolution of deformation process through an updated Lagrangian scheme. This 3-D elastoplastic finite element code allows large elastoplastic strains and rotations, following a fully implicit time integration scheme.

The configuration of the sheet, tools and the state variables are updated at the end of each time increment $\Delta \mathrm{t}$, and the new configuration is taken as reference for the subsequent time step. To calculate an approximate first solution for incremental displacements, stresses and frictional contact forces is used an explicit approach. Because this first solution satisfies neither the variational principle for the coherence condition, due to the strong non-linearities of the problem, a fully- implicit algorithm of Newton-Raphson type is used for the correction of the first approximation at instant $t+\Delta t$.

DD3IPM models the geometry of the forming tools by parametric Bézier surfaces or Nagata patches [5]. Contact is established between the deformable body (blank sheet) and the tools, which are assumed to behave rigidly. The frictional contact between the two bodies is interpreted with a classical Coulomb's law with an augmented Lagrangian approach. The numerical schemes follow a frictional contact algorithm that operates directly on the parametric Bézier surfaces or the Nagata patches [6].

\subsection{Constitutive Models}

Several efforts have been done by scientific community to improve and create new constitutive models in finite element codes. These efforts are now providing a more efficient description of the mechanical behaviour of sheet metals [7]. Developments are achieved through the implementation of new and more accurate yield criteria associated to the isotropic and kinematic hardening behaviour. The orthotropic yield criterion is currently used to model the planar anisotropy of the blank. In turn, the isotropic and kinematic hardening describes the evolution of the flow stress and the yield surface with the plastic work.

The study of the kinematic behaviour is essential in case of Bauschinger effect. This effect represents the material's answer to the multiple tension-compression cycles to which material points are submitted during the forming process.

In the present work it is used a Voce law to describe the isotropic work hardening since it is the law that better describes the mechanical behaviour of the aluminum alloy used in the proposed geometry. The equation of the mentioned law is given by:

$$
Y=Y_{0}+R_{\text {sat }}\left[1-\exp \left(-C_{Y} \bar{\varepsilon}^{p}\right)\right]
$$

where $R_{\text {sat }}$ and $C_{Y}$ are material parameters, and $\bar{\varepsilon}^{p}$ is the equivalent plastic strain. $Y_{0}$ is the initial yield stress and $Y$ is the yield stress.

Kinematic hardening is computed by Lemaître \& Chaboche evolution law, which is defined by:

$$
\dot{\mathbf{X}}=C_{x}\left[\frac{X_{s a t}}{\bar{\sigma}}\left(\boldsymbol{\sigma}^{\prime}-\mathbf{X}\right)-\mathbf{X}\right] \dot{\bar{\varepsilon}}^{p}, \mathbf{X}(0)=0
$$

being $C_{x}$ and $X_{\text {sat }}$ the material parameters. $\mathbf{X}$ is the back stress tensor, $\boldsymbol{\sigma}^{\prime}$ the deviatoric Cauchy stress tensor and 
$\bar{\sigma}$ the equivalent tensile stress as computed from the isotropic von Mises yield criterion.

For this case study, it is intended to manufacture the multimedia system chassis in aluminum alloy, in the grade AA5182-O [7]. For this specific material, the constitutive parameters to be used in equations 1 and 2 are depicted on Table 1.

Table 1. Material parameters of Voce with Kinematic Hardening law for AA5181-O.

\begin{tabular}{|c|c|c|c|c|c|}
\hline & $\mathrm{Y}_{0}$ & $\mathrm{C}_{\mathrm{R}}$ & $\mathrm{R}_{\text {sat }}$ & $\mathrm{C}_{\mathrm{X}}$ & $\mathrm{X}_{\text {sat }}$ \\
\hline $\begin{array}{l}\text { AA5182-O } \\
\text { (Hill'48) }\end{array}$ & 148.5 & 9.7 & 192.4 & 152.7 & 26.0 \\
\hline
\end{tabular}

\subsection{Elastic properties evolution}

The majority of studies of sheet metal forming considers the elastic behavior as isotropic and constant, assuming that plastic work has no effect in the elastic properties. In this connection, it is considered that when a permanent plastic deformation takes place, upon unloading, the elastic strain will be recovered. Thereby the unloading line is parallel to the initial elastic loading line. Other studies claim that the elastic properties can evolve with the plastic work, assuming that the elastic behavior remains isotropic but not constant with the plastic deformation [8-11].

Currently there are no consensus in which models have higher influence in the prediction of materials' behavior. The accuracy of each study is defined through the mathematical models implemented and depends on the studied material. Alves et. al [10] point out that for some materials, taking into account the evolutionary isotropic elasticity can have a larger effect on springback prediction than taking into account the kinematic hardening. This results were plainly observed for the A6016-T4 aluminum alloy. Moreover, in case of DP600 steel, the influence is almost similar. Zang et. al [8] arrived to similar conclusions, refereeing that for DP780 steel, the unloading modulus is more important than Bauschinger effect in springback prediction.

In finite element codes, mechanical behavior is described in a macro approach based on phenomenological laws. The numerical tools consider different behavior laws of the elastic properties and their variation with the equivalent plastic strain.

Several studies referred an inelastic recovery behavior after plastic deformation. Such behavior was already proved for several metals, such as copper, some aluminum alloys and some ferrous alloys. With this study exists the possibility of being demonstrated the evolutionary isotropic elasticity of the materials. This results will allow a more accurate performance prediction of the developed component.

$\mathrm{Yu}$ [12] referred the elastic modulus variation with plastic strain as a macroscopic phenomenon. However, to this author, from the microscopic view this condition could be inducted by microstructural evolution, textural change and internal stress during plastic deformation. Other authors, as Yang et al. [8,9] also adopted microscopic and macroscopic measurements and concluded that movement of mobile dislocation and dislocation pileup are the main sources for the decrease of the elastic modulus.

In the present study, only macroscopic evaluations will be considered because the goal is to realize how this variation affects the component performance and not why it does it.

The evolution of Young modulus as a function of the plastic strain is given in this work throughout the law proposal by Alves et. al. [10] This law is based on the experimental work of Yang et $\mathrm{Al}[8,9]$, who proposed an evolution law based on experimental measurements given by a polynomial equation, however its interval of validity is restricted to less than $25 \%$ of plastic strain. In sheet metal forming the maximum equivalent plastic strain is usually higher than $25 \%$, and because of that Alves et. al. introduced an exponential equation with saturation which describes better the evolution of the elastic properties in a wider range of plastic strains. The evolution law was implemented in DD3IMP FE code and it is given in a generic form by:

$$
E=E_{0}+k_{E} \cdot E_{0}\left[1-\exp \left(-C_{E} \bar{\varepsilon}^{p}\right)\right]
$$

where $E_{0}$ is the value of the initial Young modulus, $k_{E}$ and $C_{E}$ are the parameters of the evolution law and $\bar{\varepsilon}^{p}$ is the equivalent plastic strain. $k_{E} \in[0,1]$ defines the saturation value of the Young modulus, $\lim _{\bar{\varepsilon} p \rightarrow+\infty}\left(\frac{E_{0}-E\left(\bar{\varepsilon}^{p}\right)}{E_{0}}\right)=k_{E}$, and $C_{E}$ measures the rate of approximation to the saturation value. Figure 2 shows a variation of Young modulus as a function of the equivalent plastic strain given by the exponentiallybased evolution law introduced by Alves et. al. [10] in equation 3. For this case, $k_{E}=0.25$ and $C_{E}=12.5$.

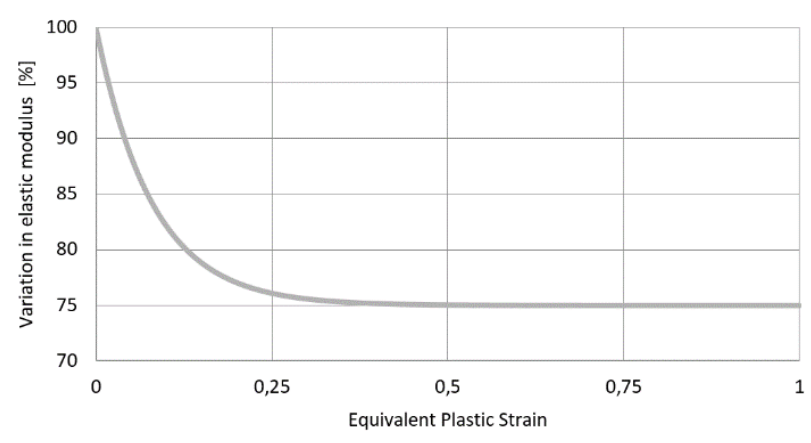

Figure 2. A variation of Young modulus as a function of the equivalent plastic strain given by the exponentially-based evolution law introduced by Alves et. al and implemented in DD3IMP FE solver.

\section{Numerical results}

\subsection{Simulation Setup}

The numerical study was separated in two main stages. In the first stage the stamping process was numerically simulated. This process is responsible for the major geometrical changes and plastic work field in the raw sheet metal. A generic scheme of the stamping tooling as 
well the general positioning of the sheet and tools in the first step is shown in Figure 3.

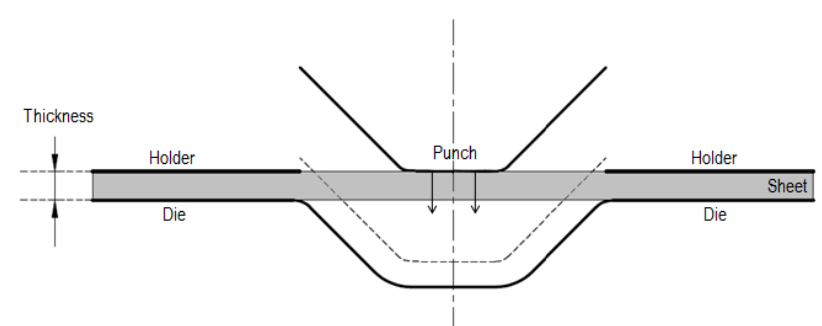

Figure 3. Schematic diagram of the stamping tools as well the general positioning of the sheet and tools at the begging of the forming step.

In the first step a blank sheet with a rectangular shape of $30 \mathrm{~mm} \times 30 \mathrm{~mm} \times 1.2 \mathrm{~mm}$ is positioned between the holder and the die. The punch moves vertically giving shape to the sheet metal, and when the punch meets to the final position it moves back in the opposite direction, allowing the springback of formed part.

The final geometry obtained in this simulation, as well of the state variables (stress and backstress tensors, equivalent plastic strain, ...) will be used as an input data to create the FE mesh for the second stage simulation. The equivalent stress and strain generated during the manufacturing process are remapped into this new FE mesh, allowing the simulation of the operating conditions taking into account, or not, the full history of the manufacturing process. In more detail, a new FE mesh is generated considering both the final geometry of the forming stage, i.e. the first stage, and the trimming geometry to obtain the new FE mesh corresponding to the geometry of the sheet formed spring as shown in Figure 1b). After, the values of the state variables are remapped from the original FE mesh to the new one. It is worth noting that the local effects of the trimming operation are not taken into account.

The second stage simulates the operating conditions of the proposed geometry, in order to investigate the role of the elastic properties evolution on the mechanical behavior of the sheet formed spring. A scheme with the general positioning of the sheet formed spring and the so-called tools used in the simulation of operating conditions is shown in Figure 4. In this stage, the sheet formed spring is loaded by a tool representing the printed circuit board. As boundary conditions, a displacement of $1 \mathrm{~mm}$ is imposed to the tool, and the reaction force is evaluated.

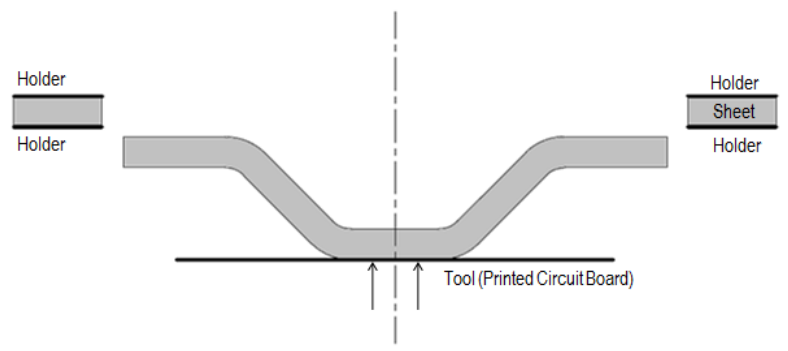

Figure 4. Schematic diagram of the sheet formed spring and the tool, i.e. the printed circuit board, as well the general positioning of the sheet formed spring and tool in the so-called working conditions (sheet formed spring being loaded).
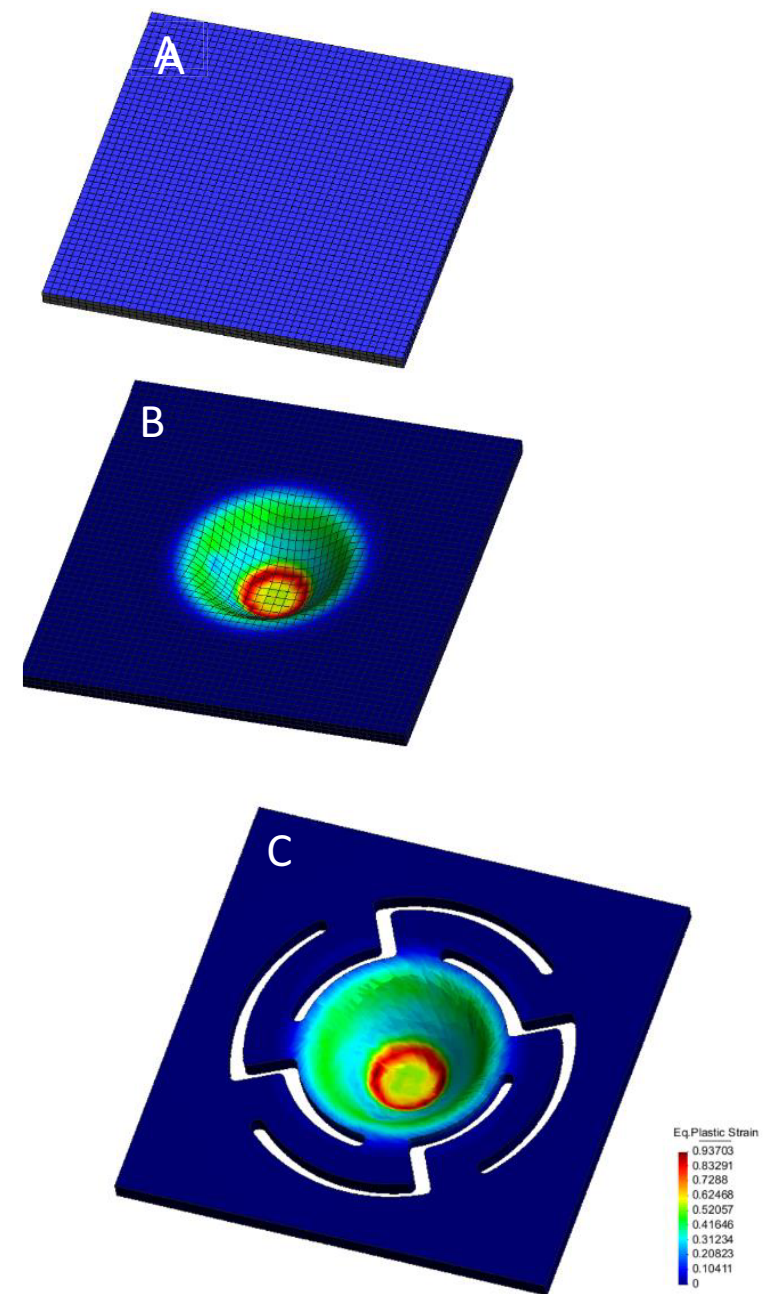

Figure 5. Mesh geometry for the two-stage simulation: a) Initial FE mesh; b) FE mesh after the stamping process and recess formation (displayed isocontour corresponds to the equivalent plastic strain); and c) FE mesh for the second stage, after trimming and remapping of the full-history state variables.

In order to precisely compare numerical results, and the role of either constitutive models or evolutive elasticity, several simulations were carried out using the same boundary conditions and FE meshes. Table 2 shows the difference between the performed simulations. There are two main types of numerical simulations: the first group only considers the assembly conditions (AC label) during the assembly of the cover in the chassis, which corresponds to pressing the sheet formed spring; the second group consider all the forming history since manufacturing process (MP label) until assembly conditions (MP.AC). For each of these groups, different material laws are used.

Table 2 shows the possible combination between first (AC) and second (MP.AC) groups with both evolutive elastic properties (EPE) and/or kinematic hardening $(\mathrm{KH})$. For example, "MP.AC.EPE.KH" is a simulation in which all condition are activated. 
Table 2. Performed numerical simulations and the difference between them in terms of material laws and pre strain information.

\begin{tabular}{|c|c|c|c|}
\hline \multicolumn{2}{|c|}{$\begin{array}{c}\text { Assembly Conditions } \\
\text { (AC) }\end{array}$} & \multicolumn{2}{|c|}{$\begin{array}{r}\text { Manufacturing Process }+ \\
\text { Assembly Conditions } \\
\text { (MP.AC) }\end{array}$} \\
\hline $\begin{array}{c}\text { Elastic } \\
\text { Properties } \\
\begin{array}{c}\text { Evolution } \\
\text { (EPE) }\end{array}\end{array}$ & $\begin{array}{c}\text { Kinematic } \\
\text { Hardening }\end{array}$ & $\begin{array}{c}\text { Elastic } \\
\text { Properties } \\
\text { Evolution } \\
\text { (EPE) }\end{array}$ & $\begin{array}{c}\text { Kinematic } \\
\text { Hardening } \\
(\mathrm{KH})\end{array}$ \\
\hline- & - & - & - \\
\hline$\checkmark$ & - & $\checkmark$ & - \\
\hline- & $\checkmark$ & - & $\checkmark$ \\
\hline$\checkmark$ & $\checkmark$ & $\checkmark$ & $\checkmark$ \\
\hline
\end{tabular}

\subsection{Results and discussion}

The results of each simulation are compared based on the reaction force of the tool named printed circuit board versus the displacement of that same tool, as shown in Figure 6. To evaluate the influence of elastic properties evolution and kinematic hardening in numerical simulation of assembly conditions (AC), Figure 6 shows the force reaction along a $1 \mathrm{~mm}$ displacement in four different cases. The four simulations were conducted taking into account the following phenomena:

- with only elastic properties evolution (AC.EPE),

- with only kinematic hardening (AC.KH),

- with both phenomena (AC.EPE.KH) and

- without any of them (AC).

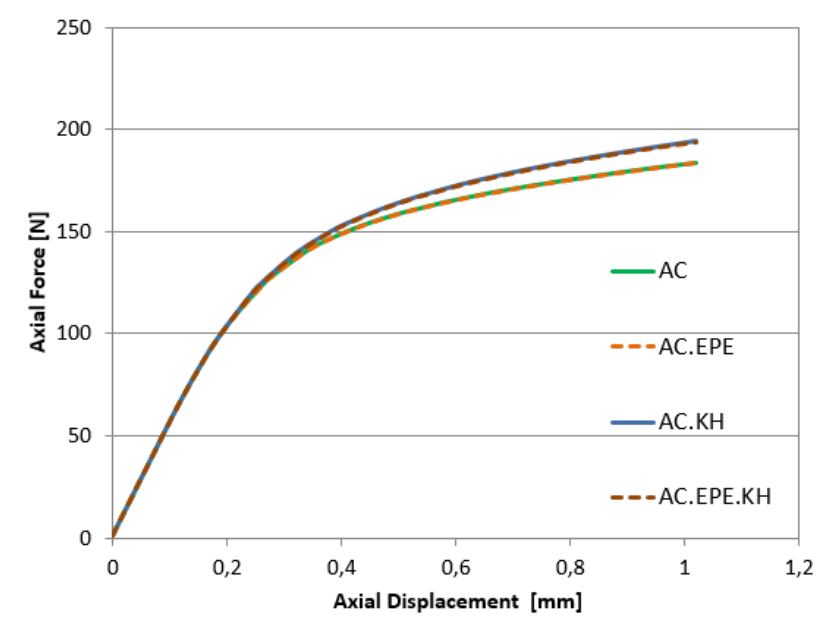

Figure 6. Curve force versus displacement of the $A C$, AC.EPE, AC.KH and AC.EPE.KH simulations during the assembling process.

In the present case, the MP condition is not activated, i.e. the forming history is not taken into account given that the state variables were not remapped from the first to the second FE mesh. In this case, the material of the sheet formed spring is considered as new material without any plastic work history.

The AC and AC.EPE curves show a very similar behavior. In the previous figure both curves have the same evolution as they are almost perfectly superposed, as well as the AC.KH and AC.EPE.KH curves which are also almost indistinguible. Therefore, this figure allows to conclude that elastic properties evolution does not influence the spring behavior. On the other hand, the results indicate that the simulations where kinematic hardening was considered displays a lower maximum force values for the same displacement of the tool (or the printed circuit board). The difference between this curves starts after around $0.2 \mathrm{~mm}$ of displacement. The maximum force is obtained in $\mathrm{AC}$ case, and was of approximately $183 \mathrm{~N}$, while for AC.KH curve was of $194 \mathrm{~N}$. This difference only represents about $6 \%$, and for the application of this sheet formed spring the value is not relevant. However, and taking into account the slope of both curves, for displacements higher than $1 \mathrm{~mm}$ this difference increase.

The evolution of the elastic properties, i.e. of the value of the Young modulus with the plastic work has almost no influence. This behavior is due to the low levels of plastic strain observed in the case where only the assembly conditions are simulated, i.e. the history is not taken into account. Table 3 shows the maximum plastic strain for the four cases. The table shows that the maximum plastic strain is consistently low. There is no substantial difference in the maximum plastic strain amongst the four different studied cases. The maximum equivalent plastic strain is of about $4.7 \%$.

Table 3. Maximum equivalent plastic strain at the end of the assembly process, for the AC, AC.EPE, AC.KH and AC.EPE.KH cases.

\begin{tabular}{|c|c|}
\hline Studied Cases & $\begin{array}{c}\text { Maximum plastic strain } \\
{[\%]}\end{array}$ \\
\hline AC & 4.7 \\
\hline AC.EPE & 4.4 \\
\hline AC.KH & 4.4 \\
\hline AC.EPE.KH & 4.6 \\
\hline
\end{tabular}

To assess the difference between the previous cases, a new set of simulations were carried out taking into consideration the stress and strain history from the manufacturing process (label MP). Figure 7 shows the force versus displacement curves of the simulations where the manufacturing process was considered.

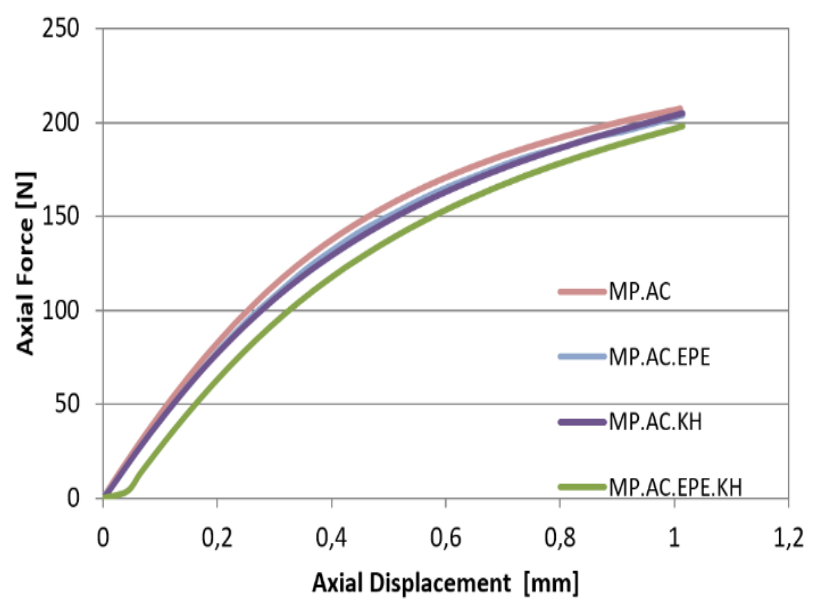

Figure 7. Curves force versus displacement of the MP.AC, MP.AC.EPE, MP.AC.KH and MP.AC.EPE.KH simulations during the assembling process. 
The results show that there is a difference between the considered cases. In the case where the material does not considers Bauschinger effect (i.e. the kinematic hardening is not taken into account) and the elastic properties evolution, the force required to perform the same displacement is about 5\% higher $(208 \mathrm{~N} / 198 \mathrm{~N})$. When the evolution of Young modulus and the Bauschinger effect are considered, the maximum force is lower, showing that these phenomena have influence in the force/displacement curve when the full loading history is considered. The higher levels of platic work developed during the manufacturing process leads to a strong variation of the elastic properties of the material.

To evaluate the influence of the manufacturing process history in the working conditions of the sheet formed spring, two simulations are compared: in the first one the deformation history from the manufacturing process was considered (MP.AC.EPE.KH) and the second one only the operating conditions was considered (AC.EPE.KH), i.e. no history. Figure 8 shows a direct comparison for these two cases, considering the obtained force versus displacement curves.

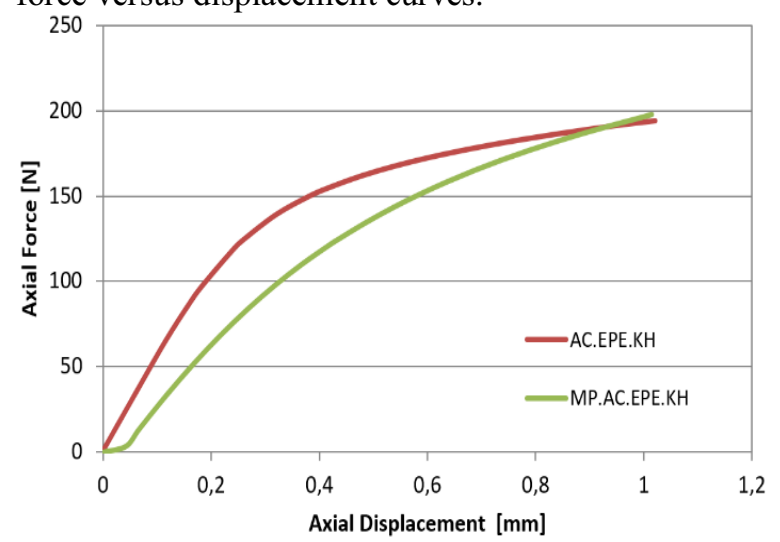

Figure 8. Curve force versus displacement of the AC.EPE.KH and MP. AC.EPE.KH simulations during assembly process.

Figure 8 indicates that there is a non-negligible difference between these two cases. For about $0.4 \mathrm{~mm}$ displacement the difference between the curves is of $22 \%(121 \mathrm{~N} / 155 \mathrm{~N})$, but AC.EPE.KH curve starts to saturate earlier and, at the end of $1 \mathrm{~mm}$ displacement, the MP.AC.EPE.KH displays a higher maximum force. The difference between the maximum force values at the end of displacement is of $3 \%(198 \mathrm{~N} / 194 \mathrm{~N})$. Although the difference, at the end of displacement is small, it could be higher if the displacement was larger, once the curves' slopes are totally different and they start to diverge at the final moments of displacement.

Figure 9 depicts the evolution of the equivalent plastic strain throughout the different manufacturing process stages and in the assembly process, with two different ranges, 0 to 0.92 and 0 to 0.044 , allowing to emphasize the deformation evolution on the tabs.

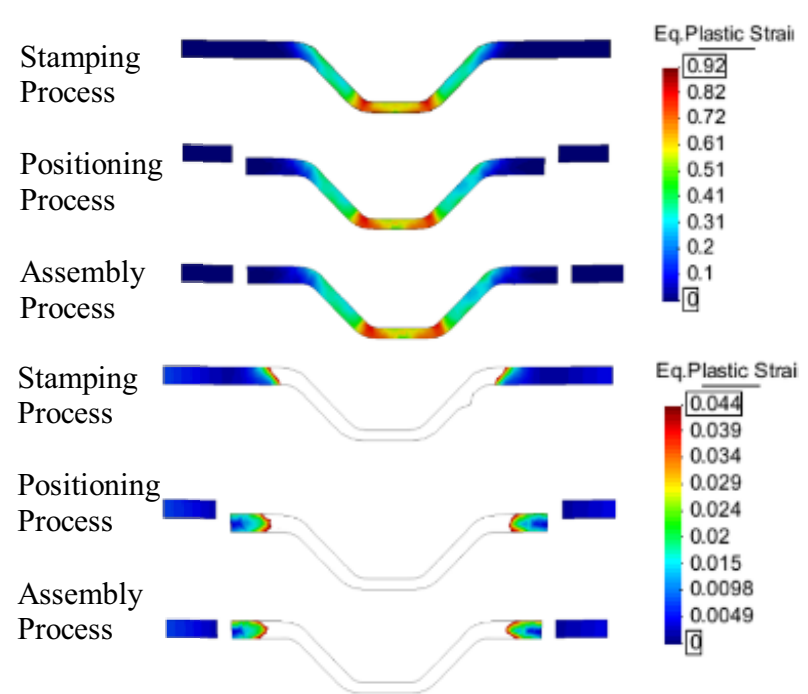

Figure 9. Evolution of the equivalent plastic strain in the investigated geometry, with two different ranges, respectively 0-0.92 and 0-0.044.

The maximum plastic strain takes place during the manufacturing process stage as expected. The plastic work caused by the remaining steps is too low when compared with the first stage. In the remaining steps, deformation is concentrated in the extremities of the tabs, and mostly in the elastic regime.

Figure 10 shows a comparison between the equivalent plastic strain for the cases presented in Figure 8.

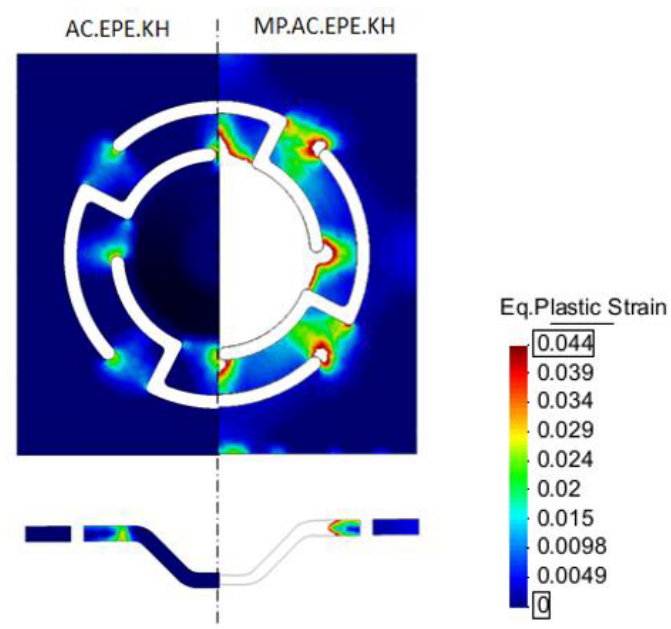

Figure 10. Comparison between the equivalent plastic strain state for the AC.EPE.KH and MP.AC.EPE.KH simulated cases. Range between 0 and 0.044 .

The region where occurs the highest plastic work is at the middle of the recess in the MP simulation, and due to the stamping process. Although the tab extremities still exhibit high levels of plastic strain. The maximum equivalent plastic strain in the tabs is between 0.30 and 0.40 , this value coincides with the point where the evolution curve do describe the evolution of the elastic properties with the plastic work starts to saturate (see Figure 1). The value of the equivalent plastic strain in the tabs is high enough to, according to the implemented law, saturate to the maximum decrease of the Young modulus. 


\section{Conclusion}

The study of the mechanical behaviour and manufacturing process of a new sheet formed spring to be applied in car multimedia industry was presented. The role of both constitutive modelling and evolutive elastic properties was analysed in order to determine their inservice conditions and specifications.

The new sheet formed spring is intended to replace several bolted connections, and thus the spring forces plays a paramount role. The analysis of the numerical results presented in the previous figures allows to conclude:

- For the investigated cases where the equivalent plastic strain is low, the evolution of the elastic properties, due to the plastic deformation, has almost no influence in the behavior of the sheet formed spring;

- The study of loading history due to the manufacturing process is important to obtain reliable results during assembling and working conditions. The effect of pre-deformation on the geometry behavior occurs, likely, due to the change of the elastic behavior of the material. A study without taking into account the loading history of the material generates different results that can lead to wrong predictions concerning the spring's behavior;

- It has been shown previously by scientific community the importance of kinematic hardening and elastic evolution properties in springback prediction of sheet metal parts. This study shows that, for the specific application studied, the role of the loading history and evolutive elastic properties is more important than the role of the kinematic hardening;

- Finally, this work demonstrates the enormous importance of a correct description of features of the mechanical behavior to accurately predict the performance of a sheet metal formed part.

\section{Acknowledgements}

This research was sponsored by:

a) Portugal Incentive System for Research and Technological Development. Project in co-promotion $n^{\circ}$ 36265/2013 (Project HMIExcel - 2013-2015), and

b) FCT with the reference project UID/EEA/04436/2013, by FEDER funds through the COMPETE 2020 - Programa Operacional Competitividade e Internacionalização (POCI) with the reference project POCI-01-0145-FEDER-006941.

\section{References}

1. D.M Neto, M.C Oliveira, J.L Alves, L.F Menezes, Influence of the plastic anisotropy modelling in the reverse deep drawing process simulation, MD, 60,368-379, (2014).

2. H Laurent, R Grèze, M.C Oliveira, L.F Menezes, P.Y Manach, J.L Alves, Numerical study of springback using the split-ring test for an AA5754 aluminium alloy, FEAD 46 51-759, (2010).

3. M.C Oliveira, J.L Alves, L.F Menezes, Algorithms and strategies for treatment of large deformation frictional contact in the numerical simulation of deep drawing process, ACME, 15, 113-162, (2008)

4. L.F Menezes, C. Teodosiu, Three dimensional numerical simulation of the deep drawing process using solid finite elements, JMPT, 97, 100-106, (2000).

5. D.M Neto, M.C Oliveira, L.F Menezes, J.L Alves, Nagata patch interpolation using surface normal vectors evaluated from the IGES file, FEAD, 72, 3546, (2013).

6. D.M Neto, M.C Oliveira, L.F Menezes, J.L Alves, Applying Nagata patches to smooth discretized surfaces used in $3 D$ frictional contact problems, CMAME, 271, 296-320, (2014).

7. L.P.M.T.M, Selection and identification of elastoplastic models for the materials used in the benchmarks, 3DS IMS, (2001).

8. M. Yang, Y. Akiama, T. Sasaki, Evaluation of change in material properties due to plastic deformation. JMPT, 151, 232-236, (2004).

9. M. Yang, Y. Akiama, T. Sasaki, Microscopic Evaluation of Change in Springback Characteristics Due to Plastic deformation, NUMIFORM, Columbus, EUA, (2004).

10. J.L Alves, M.C Oliveira, L.F Menezes, S. Bouvier, Numerical evaluation of the influence of the elastic properties evolution in springback prediction, IDDRG, 161-168, (2006).

11. R Padmanabhan, M.C Oliveira, A. Ramalho, J.L Alves, L.F Menezes, Influence of plastic work on the elastic properties of steel, AMPT, p. 22, (2009). 